\title{
Diabetic retinopathy is not present in newly diagnosed diabetic patients
}

\author{
Mayer B. Davidson ${ }^{1}$
}

Received: 7 September 2016 / Accepted: 28 September 2016 / Published online: 14 October 2016

(C) Springer-Verlag Berlin Heidelberg 2016

Keywords Diabetic retinopathy · Isolated retinopathy signs · Newly diagnosed diabetes

\section{Abbreviation \\ ETDRS Early Treatment Diabetic Retinopathy Study}

To the Editor: Ponto and colleagues would have us believe that $12.6 \%$ of type 2 diabetic patients newly diagnosed through a screening programme of the general population had retinopathy at the time of diagnosis [1]. This is not the case. They used fundus photographs that were interpreted using the criteria of the Early Treatment Diabetic Retinopathy Study (ETDRS). Those criteria for mild diabetic retinopathy are 'at least one microaneurysm, and definition not met for moderate nonproliferative retinopathy, severe nonproliferative retinopathy, early proliferative retinopathy, or high-risk proliferative retinopathy' [2]. Of the patients who screened positive for diabetes, $11.6 \%$ had mild diabetic retinopathy by these criteria [1]. It turns out that $5-10 \%$ of the general population without diabetes or hypertension have signs of isolated retinopathy that meet the ETDRS criteria for 'mild' diabetic retinopathy. Furthermore, up to $10 \%$ of people over the age of 40 years without both diabetes and isolated retinopathy signs develop these isolated retinopathy signs over 5 years, while remaining free of diabetes [3]. Indeed, Ponto and colleagues also found that $10 \%$ of age- and sex-matched controls without diabetes

Mayer B. Davidson

mayerdavidson@cdrewu.edu

1 Department of Internal Medicine, Charles R. Drew University, 1731 East 120th Street, Los Angeles, CA 90059, USA had changes that fulfilled the ETDRS criteria for mild diabetic retinopathy [4]. As a result of the issue of signs of isolated retinopathy in the general population, the cut-off point for $\mathrm{HbA}_{1 \mathrm{c}}$ levels for the diagnosis of diabetes was based on the presence of moderate diabetic retinopathy, not mild diabetic retinopathy [5]. Thus, the concept that prolonged hyperglycaemia (somehow) causes diabetic retinopathy remains intact, allowing our patients and their carers the opportunity to forestall this complication [6].

Duality of Interest The author declares that there is no duality of interest associated with this letter.

Contribution Statement The author was the sole contributor to this letter.

\section{References}

1. Ponto KA, Koenig J, Peto T et al (2016) Prevalence of diabetic retinopthy in screening-detected diabetes mellitus: results from the Gutenberg Health Study (GHS). Diabetologia 59:1913-1919

2. Early Treatment Diabetic Retinopathy Study Research Group (1991) Early treatment diabetic retinopathy study design and baseline patient characteristics: ETDRS report number 7. Ophthalmology 98:742

3. Nguyen TT, Wang JJ, Wong TY (2007) Retinal vascular changes in pre-diabetes and prehypertension: new findings and their research and clinical implications. Diabetes Care 30:2708-2715

4. Raum O, Lamparter J, Ponto KA et al (2015) Prevalence and cardiovascular associations of diabetic retinopathy and maculopathy: results from the Gutenberg Health Study. PLoS One 10, e0127188

5. The International Expert Committee (2009) International Expert Committee report on the role of the A1C assay in the diagnosis of diabetes. Diabetes Care 32:1327-1334

6. The Diabetes Control and Complications Trial Research Group (1995) The relationship of glycemic exposure $\left(\mathrm{HbA}_{1 \mathrm{c}}\right)$ to the risk of development and progression of retinopathy in the diabetes control and complications trial. Diabetes 44:968-983 